Pacific Journal of Mathematics

ON ATTACHING 3-HANDLES TO A 1-CONNECTED 


\title{
ON ATTACHING 3-HANDLES TO A 1-CONNECTED 4-MANIFOLD ${ }^{\dagger}$
}

\author{
BRUCE TRACE
}

\begin{abstract}
This paper studies various properties of 3-handle presentations of 1-connected, smooth, 4-manifolds-with-connectedboundary. Although the question of whether or not such 4-manifolds have handle presentations consisting solely of 0 -, 1- and 2-handles remains open, the main results of this paper do imply that the essential structure of such 4-manifolds is contained in a neighborhood of their 2-skeleton.
\end{abstract}

Throughout this article all maps and manifolds will be of class $C^{\infty}$. The key idea exploited here is that a knot in the boundary of a 4-manifold which is slice in this 4-manifold (i.e., which bounds a smooth, property embedded 2-disc) may be viewed as the cocore of a 2-handle - the 2-handle being a tubular neighborhood of the slice disc. Thus, if $M^{4}$ is obtained from $W^{4}$ by attaching a 3-handle, $h^{3}$, along an embedded $S^{2} \times[-1,1]$ in $\partial W^{4}$, which is denoted $W^{4} \mathbf{U}_{\Sigma^{2}}$ $h^{3}$ where $\Sigma^{2}$ is the image of $S^{2} \times\{0\}$ under this embedding, then in order to construct a 2-handle which is complementary to $h^{3}$ we need only construct a knot $K \subset \partial W^{4}$ which meets $\Sigma^{2}$ transversely in a single point (i.e., $K$ and $\Sigma^{2}$ are complementary in $\partial W^{4}$ ) and which is slice in $W^{4}$. If the boundary of $W^{4}$ is connected, the existence of a knot $K \subset \partial W^{4}$ which is complementary to $\Sigma^{2}$ is clearly equivalent to $\Sigma^{2}$ not separating $\partial W^{4}$. If $W^{4}$ is 1-connected, the following proposition tells us that a knot complementary to $\Sigma^{2}$ must be slice.

Proposition 1. Suppose $W^{4}$ is a 1-connected 4-manifold-withconnected-boundary. If $K$ is a knot in $\partial W^{4}$ which is complementary to an embedded 2-sphere $\Sigma^{2}$ in $\partial W^{4}$, then $K$ is slice in $W^{4}$.

The proof of Proposition 1 is based on the Norman trick [6], which is discussed later in the paper.

The discussion above implies.

Proposition 2. Suppose $M=W^{4} \bigcup_{\Sigma^{2}} h^{3}$ and $\widetilde{M}^{4}=W^{4} \bigcup_{\tilde{\Sigma}^{2}} \widetilde{h}^{3}$ are 1-connected 4-manifolds-with-connected-boundary. If there exists a knot $K$ in $\partial W^{4}$ such that $K$ is simultaneously complementary to both $\Sigma^{2}$ and $\widetilde{\Sigma}^{2}$, then $M^{4}$ is diffeomorphic to $\widetilde{M}^{4}$.

Standard 3-manifold techniques yield. 
Proposition 3. Suppose $W^{3}$ is a connected, orientable, 3-manifold. If $\Sigma^{2}$ and ${\widetilde{\Sigma^{2}}}^{2}$ are disjoint, nonseparating, embedded 2-spheres in the interior of $W^{3}$, then there exists a knot $K$ in $W^{3}$ which is simultaneously complementary to both $\Sigma^{2}$ and $\widetilde{\Sigma}^{2}$.

If $\Sigma^{2} \cap \widetilde{\Sigma}^{2} \neq \varnothing$, by isotoping $\Sigma^{2}$ to $\Sigma_{0}^{2}$ where $\Sigma_{0}^{2}$ meets $\widetilde{\Sigma}^{2}$ transversely, then by performing cutting and pasting techniques to innermost circles of intersection in $\Sigma_{0}^{2}$ together with an induction argument we arrive at

Proposition 4. Suppose $W^{3}$ is a connected, orientable, 3-manifold. If $\Sigma^{2}$ and $\widetilde{\Sigma}^{2}$ are nonesparating, embedded 2-spheres in the interior of $W^{3}$, then there exists a finite sequence $\Sigma_{0}^{2} \rightarrow \Sigma_{1}^{2} \rightarrow \cdots \rightarrow \Sigma_{m}^{2}=\widetilde{\Sigma}^{2}$ of nonseparating, embedded 2-spheres in the interior of $W^{3}$ such that $\Sigma_{i}^{2} \cap \Sigma_{i+1}^{2}=\varnothing$ for $i=0,1, \cdots, m-1$, and $\Sigma_{0}^{2}$ is isotopic to $\Sigma^{2}$.

Propositions 2, 3, and 4 combine to yield

Proposition 5. Suppose $M^{4}=W^{4} \bigcup_{\Sigma^{2}} h^{3}$ and $\widetilde{M}^{4}=W^{4} \bigcup_{\tilde{\Sigma}_{2}} \widetilde{h}^{3}$ are 1-connected 4-manifolds-with-connected-boundary, them $M^{4}$ is diffeomorphic to $\widetilde{M}^{4}$.

Proposition 5 together with an inductive argument are used to prove the nontrivial implication in

THEOREM 1. Suppose $M^{4}=W^{4} \bigcup_{\Sigma_{1}^{2}} h_{1}^{3} \cup \cdots \bigcup_{\Sigma_{k}^{2}} h_{k}^{3}$ and $\tilde{M}^{4}=$ $\widetilde{W}^{4} \bigcup_{\tilde{s}_{1}^{2}} \widetilde{h}_{1}^{3} \cup \cdots \cup_{\tilde{s}_{k}^{2}} \widetilde{h}_{k}^{3}$ are 1-connected, 4-manifolds-with-connectedboundary. Then $M^{4}$ is diffeomorphic to $\widetilde{M}^{4}$ if and only if $W^{4}$ is diffeomorphic to $\widetilde{W}^{4}$.

REmark. In [4] F. Laudenbach and V. Poenaru showed (essentially) that Theorem 1 remains true if we assume $\partial M^{4}=S^{3}$ rather than $\pi_{1}\left(M^{4}\right)=1$. (See [5] for details.)

By introducing $k$ complementary 2-, 3-handle pairs to $W^{4}$ then employing Theorem 1 to reattach these complementary 3 -handles so as to form $M^{4} \cup k$ (2-handles) from $W^{4} \cup k$ (complementary 2-,3-handle pairs), we observe that $M^{4}$ embeds in $W^{4}$. Hence we observe

Theorem 2. Suppose $M^{4}$ and $W^{4}$ are as in the statement of Theorem 1. Then, if $N^{4}$ is an arbitrary 4-manifold, $M^{4}$ embeds in $N^{4}$ if and only if $W^{4}$ embeds in $N^{4}$.

Theorem 1 together with the cutting and pasting argument of Proposition 4 imply 
THEOREM 3. Suppose $M^{4}$ and $W^{4}$ are as in the statement of Theorem 1, and $W^{4}=W_{1}^{4} \#_{0} W_{2}^{4}$. Then $M^{4}=M_{1}^{4} \#_{\partial} M_{2}^{4}$ where $M_{i}^{4}=$ $W_{i}^{4} \cup(3-h a n d l e s)$ for $i=1,2$.

I would like to thank R. D. Edwards for his suggestions. These suggestions served to simplify many points in the proof of Theorem 1.

Definitions and Notation. We shall assume the reader is familar with the basic definitions and results of differential topologyin particular handle theory.

We shall denote the unit ball in $R^{n}$ by $B^{n}$. Any diffeomorph of $B^{n}$ is called an $n$-disc. The $(n-1)$-sphere is denoted by $S^{n-1}$. If $M^{n}$ is an $n$-manifold-with-boundary we denote the boundary of $M^{n}$ by $\partial M^{n}$. The interior of $M^{n}$, denoted int $M^{m}$, is int $M^{n}=M^{n}-$ $\partial M^{n}$. If $X$ is a subset of $M^{n}$, then we shall use $\operatorname{cl} X$ to denote the closure of $X$ in $M^{n}$.

If $M_{1}^{n}$ and $M_{2}^{n}$ are two manifolds, then $M_{1}^{n} \# M_{2}^{n}$ denotes the (interior) connected sum of $M_{1}^{n}$ with $M_{2}^{n}$ and the boundary connected sum of $M_{1}^{n}$ with $M_{2}^{n}$ is denoted by $M_{1}^{n} \#_{0} M_{2}^{n}$.

Throughout this paper lower case " $h$ " is used only for "handle". The symbols $h^{r}, h_{i}^{r}$ and $\tilde{h}_{i}^{r}$ all represent $r$ handles. The subscript denotes the order of attaching.

An arc is an embedded 1 disc and a knot in a 3-manifold is an embedded $S^{1}$.

Proof of Proposition 1. Since $W^{4}$ is 1-connected there exists an immersed 2-disc $\Delta_{1}^{2} \subset W^{4}$ bounded by $K$. We may assume that $\Delta_{1}^{2}$ is properly immersed in $W^{4}$ (i.e. $\Delta_{1}^{2} \cap \partial W^{4}=\partial \Delta_{1}^{2}$ ) and that the singular set of $\Delta_{1}^{2}$ consists of a finite number of transverse double points. Since $\Sigma^{2}$ meets $K$ transversely in a single point, there exists an embedded 2-sphere $\Sigma_{1}^{2} \subset \operatorname{int} W^{4}$ such that $\Sigma_{1}^{2}$ meets $\Delta_{1}^{2}$ transversely in a single point, say $g$.

If $p$ is a double point of $\Delta_{1}^{2}$, let $\alpha$ be an arc in $\Delta_{1}^{2}$ joining $p$ to $g$ such that $\alpha$ meets no other double point of $\Delta_{1}^{2}$. We now apply the Norman trick. This means we pipe $\Delta_{1}^{2}$ to $\Sigma_{1}^{2}$ via a small annullus contained in the normal bundle of $\Delta_{1}^{2}$ restricted to $\alpha$. The net result of this piping operation is to cancel the double point $p$ of $\Delta_{1}^{2}$ against the point $g=\Delta_{1}^{2} \cap \Sigma_{1}^{2}$. Thus we have replaced the 2-disc $\Delta_{1}^{2}$ with a new 2-disc, $A_{2}^{2}$, which is bounded by $K$ and has one fewer double point than $\Delta_{1}^{2}$. Inductively we obtain $K$ bounds a properly embedded 2-disc, i.e., $K$ is slice in $W^{4}$.

Proof of Proposition 2. By Proposition 1, such a knot $K$ is slice 
in $W^{4}$. Let $\Delta^{2}$ be a slice disc for $K$ in $W^{4}$. If $N\left(\Delta^{2}\right)$ is a small tubular neighborhood of $\Delta^{2}$ then $N\left(\Delta^{2}\right)$ is a trivial 2-discbundle over $\Delta^{2}$. Hence, $W^{4}$ is obtained from $\mathrm{cl}\left(W^{4}-N\left(\Delta^{2}\right)\right)$ by attaching a 2-handle, namely $N\left(\Delta^{2}\right)$, where $\Delta^{2}$ is the cocore disc of $N\left(\Delta^{2}\right)$. Since $K$ is simultaneously complementary to both $\Sigma^{2}$ and $\widetilde{\Sigma}^{2}$, we obtain the 2-handle $N\left(\Delta^{2}\right)$ is complementary to each of the 3 -handle $h^{3}$ and $\widetilde{h}^{3}$. Therefore both $M^{4}$ and $\widetilde{M}^{4}$ are diffeomorphic to $\operatorname{cl}\left(W^{4}-N\left(\Delta^{2}\right)\right)$.

Proof of Proposition 3. Since neither $\Sigma^{2}$ nor $\widetilde{\Sigma}^{2}$ separate $W^{3}$ we must have that $W^{3}-\left(\Sigma^{2} \cup{\widetilde{\Sigma^{2}}}^{2}\right)$ consists of at most two components. If $W^{3}-\left(\Sigma^{2} \cup \widetilde{\Sigma}^{2}\right)$ is connected, the existence of the desired knot $K$ is obvious. If $W^{3}-\left(\Sigma^{2} \cup \widetilde{\Sigma}^{2}\right)$ consists of two components, say $C_{1}$ and $C_{2}$, choose points $p \in \Sigma^{2}$ and $\widetilde{p} \in \widetilde{\Sigma}^{2}$. Because both $W^{3}-\Sigma^{2}$ and $W^{3}-\widetilde{\Sigma}^{2}$ are connected and $C_{i}, i=1,2$, is arc connected, there exist arcs $\alpha_{i}$ properly embedded in $\operatorname{cl}\left(C_{i}\right), i=1,2$, joining $p$ to $\widetilde{p}$. Thus $K=\alpha_{1} \cup \alpha_{2}$ is the desired knot.

Proof of Proposition 4. We may isotope $\Sigma^{2}$ to $\Sigma_{0}^{2}$, where $\Sigma_{0}^{2} \cap \widetilde{\Sigma}^{2}$ consists of a finite collection of transverse, disjoint circles. Let $\sigma^{1}$ be an innermost circle of intersection in $\widetilde{\Sigma}^{2}$ bounding the innermost $\operatorname{disc} \widetilde{\Delta}^{2} \subset \widetilde{\Sigma}^{2}$, i.e., $\partial \widetilde{\Delta}^{2}=\sigma^{1}$ and $\Sigma_{0}^{2} \cap\left(\operatorname{int} \widetilde{\Delta}^{2}\right)=\varnothing$.

Let $\Delta_{1}^{2} \cup \Delta_{2}^{2}=\Sigma_{0}^{2}$, where $\Delta_{1}^{2} \cap \Delta_{2}^{2}=\sigma^{1}$. We may isotope the 2spheres $\widetilde{\Delta}^{2} \bigcup_{\sigma} \Delta_{i}^{2}$ to disjoint 2-spheres $\Sigma_{1 i}^{2}, i=1,2$, where both $\Sigma_{11}^{2}$ and $\Sigma_{12}^{2}$ meet $\widetilde{\Sigma}^{2}$ in at least one fewer component than $\Sigma_{0}^{2}$ and $\Sigma_{1 i}^{2} \cap \Sigma_{0}^{2}=$ $\varnothing, i=1,2$. Since the connected sum of $\Sigma_{11}^{2}$ with $\Sigma_{12}^{2}$ gives back $\Sigma_{0}^{2}$ (up to isotopy), we must have either $\Sigma_{11}^{2}$ or $\Sigma_{12}^{2}$ not separating $W^{3}$. Let $\Sigma_{1}^{2}$ be this noseparating 2 sphere.

Since $\Sigma_{1}^{2}$ meets $\widetilde{\Sigma}^{2}$ in fewer components than does $\Sigma_{0}^{2}$, we may inductively continue the process until we come to a 2 -sphere which is disjoint from $\widetilde{\Sigma}^{2}$. Having done so, we will have constructed the desired sequence of 2 -spheres.

Proof of Proposition 5. Since both $M^{4}$ and $\widetilde{M}^{4}$ have connected boundary, it follows that $\Sigma^{2}$ and $\widetilde{\Sigma}^{2}$ are nonseparating 2-spheres in the connected, orientable 3-manifold $\partial W^{4}$. Thus, by Proposition 4, there exists a 2-sphere $\Sigma_{0}^{2} \subset \partial W^{4}$ which is isotopic to $\Sigma^{2}$ and a finite sequence of nonseparating 2 -spheres $\Sigma_{0}^{2} \rightarrow \Sigma_{1}^{2} \rightarrow \cdots \rightarrow \Sigma_{m}^{2}=\widetilde{\Sigma}^{2}$ where $\Sigma_{i}^{2} \cap \Sigma_{i+1}^{2}=\varnothing, i=0,1, \cdots, m-1$. By Proposition 3 there exists a knot $K_{i} \subset \partial W^{4}$ such that $K_{i}$ is simultaneously complementary to both $\Sigma_{i}^{2}$ and $\Sigma_{i+1}^{2}$ for $i=0,1, \cdots, m-1$.

Set $M_{i}^{4}=W^{4} \mathbf{U}_{\Sigma_{i}^{2}} h_{i}^{3}$ for $i=0,1, \cdots, m-1$. Since $\Sigma^{2}$ is isotopic to $\Sigma_{0}^{2}$, we have $M^{4}$ is diffeomorphic to $M_{0}^{4}$. We apply Proposition 2 to obtain $M_{i}^{4}$ is diffeomorphic to $M_{i+1}^{4}, i=0, \cdots, \cdots, m, 1$. Thus $M^{4}$ is diffeomorphic to $\widetilde{M}^{4}$. 
Proof of Theorem 1. Suppose $f: M^{4} \rightarrow \widetilde{M}^{4}$ is a diffeomorphism. Since the cocore of 3 -handle is 1-dimensional and $M^{4}$ is simply connected, there exists an ambient isotopy of $\widetilde{M}^{4}, F_{t}$, such that $F_{0}$ is the identity and $F_{1}\left[f\right.$ (cocore of $\left.\left.h_{i}^{3}\right)\right]=$ (cocore of $\left.\tilde{h}_{i}^{3}\right), i=1, \cdots, k$. Then both $F_{1}\left(f\left(h_{i}^{3}\right)\right)$ and $\tilde{h}_{i}^{3}$ are tubular neighborhoods of the cocore of $\widetilde{h}_{i}^{3}$, $i=1, \cdots, k$. Therefore there exists an isotopy $G_{t}$ of $\tilde{M}^{4}$ such that $G_{0}$ is the identity and $G_{1}\left(F_{1}\left(f\left(h_{i}^{3}\right)\right)\right)=\widetilde{h}_{i}^{3}, \quad i=1, \cdots, k$. Set $g=$ $G_{1} \circ F_{1} \circ f: M^{4} \rightarrow \widetilde{M}^{4}$. Then $g \mid W^{4}$ is a diffeomorphism of $W^{4}$ onto $\widetilde{W}^{4}$.

As previously stated, the nontrival implication in Theorem 1 proceeds via induction on $k$. Let $g: W^{4} \rightarrow \widetilde{W}^{4}$ be a diffeomorphism assume $k=1$. Set $g\left(M^{4}\right)=\widetilde{W} \bigcup_{g\left(\Sigma_{1}^{2}\right)} h^{3}$. Then $g$ induces a diffeomorphism of $M^{4}$ onto $g\left(M^{4}\right)$. Therefore $g\left(M^{4}\right)$ and $\widetilde{M}^{4}$ satisfy the hypothesis of Proposition 5. This yields $g\left(M^{4}\right)$ is diffeomorphic to $\widetilde{M}^{4}$ and hence the theorem is true for $k=1$.

Set

$$
W_{*}^{4}=W^{4} \bigcup_{\Sigma_{1}^{2}} h_{1}^{3} \cup \cdots \sum_{\Sigma_{k-1}^{2}}^{\bigcup} h_{k-1}^{3} \quad \text { and } \quad \widetilde{W}_{*}^{4}=\widetilde{W}_{\widetilde{\Sigma}_{1}^{4}} \bigcup \widetilde{h}_{1}^{3} \cup \cdots \widetilde{\Sigma}_{k-1}^{2} \widetilde{h}_{k-1}^{3} .
$$

By induction, if $W^{4}$ is diffeomorphic to $\widetilde{W}^{4}$ then $W_{*}^{4}$ is diffeomorphic to $\widetilde{W}_{*}^{4}$. By repeating the argument used in the $k=1$ case to $M^{4}=$ $W_{*}^{4} \bigcup_{\Sigma_{1}^{2}} h_{k}^{3}$ and $\widetilde{M}^{4}=\widetilde{W}_{*}^{4} \bigcup_{\widetilde{\Sigma}_{k}^{2}} \widetilde{h}_{k}^{3}$ we obtain $M^{4}$ is diffeomorphic to $\widetilde{M}^{4}$ which completes the proof of Theorem 1.

Proof of Theorem 2. Let $\Delta^{3} \subset \partial W^{4}$ be a 3-cell disjoint from $\mathrm{U}_{i=1}^{k} \sum_{i}^{2}$. Attach $k$ complementary 2-,3-handle pairs to $W^{4}$ with attaching tubes meeting $\partial W^{4}$ in the interior of $\Delta^{3}$. Observing that $W^{4} \cup k$ (2-handles) is simply connected if $W^{4}$ is simply connected, we apply Theorem 1 to alter the fashion in which 3 -handles are attached to $W^{4} \cup k$ (2-handles). We reattach the 3 -handle which initially is complementary to the $i$ th 2 -handle to $\Sigma_{i}^{2}$.

Since $W^{4}$ union $k$ complementary 2 -,3-handle pairs is diffeomorphic to $W^{4}$ upon altering the fashion in which 3 -handles are attached to $W^{4} \cup\left(2\right.$-handles), we have that $W^{4}$ is diffeomorphic to $M^{4} \cup k$ (2-handles). Hence any embedding of $W^{4}$ into $N^{4}$ yields an embedding of $M^{4}$ into $N^{4}$.

The converse direction is trivial.

Proof of Theorem 3. Let $\Sigma^{2} \subset \partial W^{4}$ be the 2-sphere along which we connect sum $\partial W_{1}^{4}$ to $\partial W_{2}^{4}$ to obtain $\partial W^{4}$. We may isotope the $\Sigma_{i}^{2}, i=1, \cdots, k$, so that they meet $\Sigma^{2}$ transversely in a finite collection of circles. Proceeding as in Proposition 4, the collection $\sum_{i}^{2}, i=1$, $\cdots, k$, may be replaced with another collection of 2 -spheres $\widetilde{\Sigma}_{i}^{2}, i=$ $1, \cdots, k$ such that $\widetilde{\Sigma}_{i}^{2} \cap \Sigma^{2}=\varnothing$ for $i=1, \cdots, k$, and $\partial W^{4}-\left(\bigcup_{i=1}^{k} \widetilde{\Sigma}_{i}^{2}\right)$ is connected. 
Set $\widetilde{M}^{4}=W^{4} \bigcup_{\tilde{\Sigma}_{k}^{2}} \widetilde{h}_{1}^{3} \cup \cdots \bigcup_{\widetilde{\Sigma}_{k}^{2}} \widetilde{h}_{k}^{3}$ and observe that $M^{4}$ and $\widetilde{M}^{4}$ satisfy the hypothesis of Theorem 1 . Hence $M^{4}$ is diffeomorphic to $\widetilde{M}^{4}$. Since $\widetilde{\Sigma}_{i}^{2} \cap \Sigma^{2}=\varnothing$ for $i=1, \cdots, k$, we have $\widetilde{M}^{4}=\widetilde{M}_{1}^{4} \#_{\partial} \widetilde{M}_{2}^{4}$ where $\widetilde{M}_{i}^{4}=\widetilde{W}_{i}^{4} \cup(3$-handles $), i=1,2$. This implies the desired result.

Some Corollaries.

CoROllary 1. Suppose $M^{4}=B^{4} \cup k$ (2-handles) $\cup k$ (3-handles) $\cong p t$. Then $M$ smoothly embeds in $S^{4}$ if and only if the link along which the 2-handles are attached to $B^{4}$ is strongly slice in $B^{4}$.

Sketch of Proof. Since $M^{4}$ is contractible, each 2-handle is attached to $B^{4}$ via 0 -framing. We leave as an exercise the fact that $B^{4} \cup k$ (2-handles) smoothly embed in $S^{4}$ if and only if the link along which 2 -handles are attached to $B^{4}$ is strongly slice, i.e., each component is slice and the slice discs can be chosen to be mutually disjoint, and each 2 -handle is attached via 0 -framing. Theorem 2 implies $M^{4}$ smoothly embeds in $S^{4}$ if $B^{4} \cup k$ (2-handles) does.

Corollary 2. Suppose $M^{4}=B^{4} \cup_{K}$ (2-handle) $\cup(3$-handle $) \cong p t$. Then the punctured double of $M^{4}$ smoothly embeds in $B^{4} \bigcup_{K}$ (2-handle). Hence, Punct $\left(D M^{4}\right)$ smoothly embeds in $S^{4}$ if and only if $K$ is slice.

Proof. Write $M^{4}=B^{4} \bigcup_{K} h^{2} \cup h^{3}$. Then $\operatorname{Punct}\left(D M^{4}\right)=B^{4} \mathbf{U}_{K}$ $h^{2} \cup h^{3} \cup\left(h^{3}\right)^{*} \cup\left(h^{2}\right)^{*}$, where $\left(h^{3}\right)^{*}$ is a 1-handle dual to $h^{3}$ and $\left(h^{2}\right)^{*}$ is a 2-handle dual to $h^{2}$.

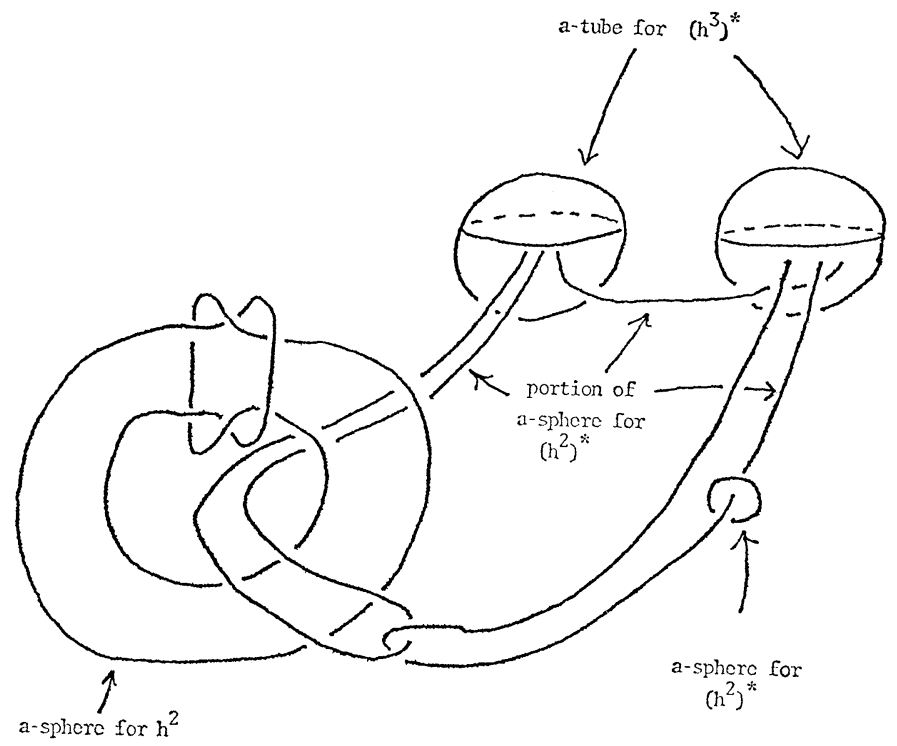

Figure 1 
We attach a 2-handle, $\left(h^{2}\right)_{m}^{*}$, to $\operatorname{Punct}\left(D M^{4}\right)$. This 2-handle is attached via 0 -framing along a meridian of the attaching sphere of $\left(h^{2}\right)^{*}$, see Figure 1.

Then by sliding $h^{2}$ over $\left(h^{2}\right)_{m}^{*}$ we can untangle the $a$-spheres of $h^{2}$ and $\left(h^{2}\right)^{*}$. Since $M^{4}$ is contractible, we must have $\left(h^{2}\right)^{*}$ algebraically cancelling $\left(h^{3}\right)^{*}$. By sliding $\left(h^{2}\right)^{*}$ over $\left(h^{2}\right)_{m}^{*}$ we can arrange that $\left(h^{2}\right)^{*}$ geometrically cancels $\left(h^{3}\right)^{*}$. Thus, Punct $\left(D M^{4}\right) \subset B^{4} \bigcup_{K} h^{2} \cup h^{3} \cup\left(h^{2}\right)_{m}^{*}$. Since the $a$-sphere of $h^{2}$ and the $a$-sphere of $\left(h^{2}\right)_{m}^{*}$ lie in disjoint 3-cells of $\partial B^{4}$ and since the $a$-sphere for $\left(h^{2}\right)_{m}^{*}$ is the unknot-we may apply Theorem 1 so as to reattach $h^{3}$ so that it geometrically cancels $\left(h^{2}\right)_{m}^{*}$. Thus Punct $\left(D M^{4}\right) \subset B^{4} \mathrm{U}_{K} h^{2}$.

Final Remarks. (1) In [3], R. Kirby and P. Melvin observed that $K$ as in Corollary 2 is slice if $\partial M=S^{3}$.

(2) Recently, S. Akbulut and R. Kirby have obtained a $M^{4}=$ $B^{4} \cup 2$ (2-handles) $\cup 2$ (3-handles) $\cong p t$. where the link along which 2-handles are attached is not known to be slice, (see [1] and [2]).

\section{REFERENCES}

1. S. Akbulut and R. Kirby, An Exotic Involution of $S^{4}$, Topology, 18 (1979), 75-81.

2. S. Akubulut and R. Kirby, to appear.

3. R. Kirby and P. Melvin, Slice Knots and Property R, Inventiones Math., 45 (1978), 57-59.

4. F. Laudenbach and V. Poenaru, $A$ Note on 4-Dimensional Handlebodies, Bull. Soc. Math., France, 100 (1972), 337-347.

5. J. Montesinos, Heegard Diagrams For 4-Manifolods, Preprint.

6. R. Norman, Dehn's Lemma for Certain 4-Manifolds, Inventiones Math., 7 (1969), 143-147.

Received October 13, 1980. Research partially supported by NSF grant MCS 7606903.

University of CALIFornia

LOS ANGELES, CA 920024

Present Address: University of Utah

Salt Lake City, UT 84112 



\section{PACIFIC JOURNAL OF MATHEMATICS}

\section{EDITORS}

DONALD BABBITT (Managing Editor)

University of California

Los Angeles, California 90024

\section{Hugo Rossi}

University of Utah

Salt Lake City, UT 84112

C. C. MOore and Arthur AGuS

University of California

Berkeley, CA 94720
J. DugundJI

Department of Mathematics University of Southern California Los Angeles, California 90007

R. FinN and J. Milgram Stanford University Stanford, California 94305

ASSOCIATE EDITORS
R. ARENS
E. F. BECKENBACH
B. H. NeumanN
F. WOLF
K. YOSHIDA

\section{SUPPORTING INSTITUTIONS}

UNIVERSITY OF ARIZONA

UNIVERSITY OF BRITISH COLUMBIA

CALIFORNIA INSTITUTE OF TECHNOLOGY

UNIVERSITY OF CALIFORNIA

MONTANA STATE UNIVERSITY

UNIVERSITY OF NEVADA, RENO

NEW MEXICO STATE UNIVERSITY

OREGON STATE UNIVERSITY
UNIVERSITY OF OREGON

UNIVERSITY OF SOUTHERN CALIFORNIA

STANFORD UNIVERSITY

UNIVERSITY OF HAWAII

UNIVERSITY OF TOKYO

UNIVERSITY OF UTAH

WASHINGTON STATE UNIVERSITY

UNIVERSITY OF WASHINGTON 


\section{Pacific Journal of Mathematics}

Vol. 99, No. $1 \quad$ May, 1982

Mariano Giaquinta, Jindrich Necas, O. John and J. Stará, On the

regularity up to the boundary for second order nonlinear elliptic systems . . 1

Siegfried Graf, Realizing automorphisms of quotients of product $\sigma$-fields . . 19

Alfred Washington Hales and Ernst Gabor Straus, Projective colorings . . . 31

Sandra Hayes, The weak Nullstellensatz for finite-dimensional complex

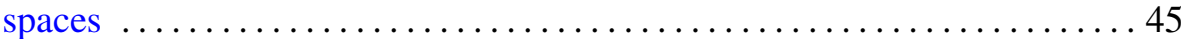

Gerald Norman Hile and Murray Harold Protter, The Cauchy problem

and asymptotic decay for solutions of differential inequalities in Hilbert

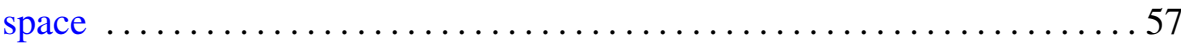

Robert D. Little, Projective space as a branched covering with orientable

branch set ......................................... 89

Jaroslav Mach, On the proximinality of Stone-Weierstrass subspaces . . . . . 997

John C. Morgan, II, On product bases ...................... 105

K. Balakrishna Reddy and P. V. Subrahmanyam, Altman's contractors

and fixed points of multivalued mappings . .................. 127

James Ted Rogers Jr., Decompositions of homogeneous continua . . . . . . . 137

Ahmed Ramzy Sourour, Characterization and order properties of

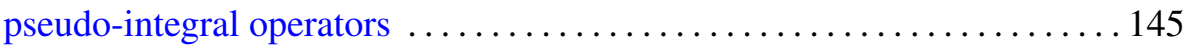

Robert Moffatt Stephenson Jr., Pseudocompact and Stone-Weierstrass

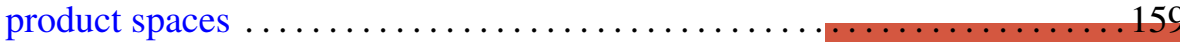

Bruce Stewart Trace, On attaching 3-handles to a 1-connected

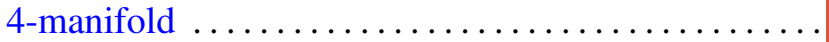

Akihito Uchiyama, The construction of certain BMO functions and the corona problem

Thomas Alva Whitehurst, An application of orthogonal polynomials to random walks ..............................

David J. Winter, Root locologies and idempotents of Lie and nonassociative algebras

William Robin Zame, The classification of uniform algebras on plane domains 